\title{
In Situ Laser-Laser carbon and oxygen isotopes measurements in carbonates: A step forward field isotopic characterization
}

\author{
DR. CHRISTOPHE THOMAZO ${ }^{1}$, PIERRE SANS-JOFRE ${ }^{2}$, \\ OLIVIER MUSSET $^{3}$, THÉOPHILE COCQUEREZ ${ }^{3}$ AND \\ STEFAN V. LALONDE ${ }^{4}$ \\ ${ }^{1}$ Laboratoire Biogéosciences, UMR CNRS 6282, Université de \\ Bourgogne Franche-Comté \\ ${ }^{2}$ IMPMC, CNRS, Sorbonne Université, MNHN \\ ${ }^{3}$ Université de Bourgogne \\ ${ }^{4}$ CNRS-UMR6538 Laboratoire Géosciences Océan \\ Presenting Author: christophe.thomazo@u-bourgogne.fr
}

Stable isotope ratios $\left(\mathrm{d}^{13} \mathrm{C}\right.$ and $\left.\mathrm{d}^{18} \mathrm{O}\right)$ of carbonates archived in the rock record are routinely used to reconstruct paleotemperatures and the secular evolution of the biogeochemical carbon cycle through Earth History. The state-of-the-art technique, employed since the mid 20th century, to measure these isotopic ratios includes: micro-drilling and/or sawing and crushing, $\mathrm{CO}_{2}$ release by wet acid digestion, gas equilibration, purification, and transfer, before gas phase IRMS measurements. While these steps are time- and resource-consuming, they provide accurate measurements from rock samples.

This study presents a new protocol involving a laser calcination system that decreases drastically the analysis time by reducing the number of preparation steps while offering the possibility of performing punctual in-situ analyses at the $\mathrm{mm}$ scale. This original method is based on the use of a fiber laser diode device emitting in near infrared at $880 \mathrm{~nm}$ and inducing the decomposition of calcium carbonate into lime and carbon dioxide.

We analyzed 9 different types of carbonates encompassing a range of isotopic compositions, between -18.2 and +3.3 and between -1.7 and $-14.6 \%$ for $\mathrm{d}^{13} \mathrm{C}_{\text {carb }}$ and $\mathrm{d}^{18} \mathrm{O}_{\text {carb }}$ VPDB, respectively. A comparison of isotopic results was performed between the classic (micro-drilling and acid digestion) vs. laser calcination methods and using both a magnetic sector IRMS and laser-based Isotope Ratio Infrared Spectrometer. The resulting isotopic cross-calibration shows a direct positive co-variation between both methods with a correlation coefficient of 0.99 and a regression slope of 1 within uncertainties for $\mathrm{d}^{13} \mathrm{C}_{\text {carb }}$ values. The $\mathrm{d}^{18} \mathrm{O}_{\text {carb }}$ values also compared well, with a correlation coefficient of 0.96 , suggesting a constant gas-solid phase isotopic equilibrium. The reproducibility of our laser calcination method shows a 1 sstandard deviation of 0.31 and 0.77 for $\mathrm{d}^{13} \mathrm{C}_{\text {carb }}$ and $\mathrm{d}^{18} \mathrm{O}_{\text {carb }}$, respectively.

We demonstrate that (i) laser calcination gives accurate and reproducible $\mathrm{C}$ and $\mathrm{O}$ isotope characterizations of carbonates, (ii) the physical effect during calcination does not introduce sensible isotopic fractionation for $\mathrm{C}$ and is accompanied by a constant isotopic equilibrium offset for $\mathrm{O}$. These findings pave the way for a new range of possibilities for carbonate isotopic measurements using rapid, punctual and easy to manipulate laser 